\title{
Desarrollo humano y desigualdad en México
}

\section{Human Development and Inequality in Mexico}

DOI: $10.32870 /$ mycp.v8i22.573

\author{
Teodoro Aguilar Ortega ${ }^{1}$
}

\begin{abstract}
Resumen
El modelo neoliberal mexicano y su política de sustentar la competitividad en el bajo costo de la mano de obra han provocado una excesiva concentración del ingreso en las esferas más altas de la población mexicana, además de creciente pobreza en nuestro país; sin embargo, las carencias no se distribuyen por igual, pues la desigualdad se expresa en estos momentos en inequidades territoriales y sociales que, a su vez, son un freno al crecimiento económico a largo plazo. Estas desigualdades se pueden corroborar al observar los muy distintos niveles de desarrollo alcanzado por las entidades de nuestro país, medido a través del índice de desarrollo humano. Si bien México ha sido por tradición una nación de desigualdades, hasta mediados de los años ochenta del siglo pasado
\end{abstract}

dichas desigualdades venían disminuyendo; sin embargo, el cambio de modelo económico ha provocado un incremento en esas inequidades. En ese sentido, el objetivo de este trabajo es conocer cómo la concentración de la riqueza ha provocado enormes niveles de desigualdad en México. La metodología utilizada es la comparación entre el nivel de desarrollo humano entre las entidades federativas de nuestro país y la evolución del índice de Gini, para entender cómo ha evolucionado la desigualdad y en qué regiones se ha concentrado la pobreza. Con ello se refuerza el argumento de que el modelo neoliberal es el causante del crecimiento de la pobreza y la desigualdad en México.

Palabras claves: pobreza, ingreso, población, inequidad, desarrollo

Artículo recibido el 18 de enero de 2018 y dictaminado el 25 de junio de 2018.

1. Universidad Nacional Autónoma de México (UNAM), Unidad Académica de Estudios Regionales (UAER). Av. Lázaro Cárdenas, Felícitas del Río s/n, Centro, C.P. 59510 Jiquilpan de Juárez, Michoacán, México. ORCID: http://orcid.org/0000-0003-3712-3787. Correo electrónico: teo_aguilar@ humanodades.unam.mx 


\section{Summary}

The Mexican neoliberal model and its policy of sustaining competitiveness in the low cost of labor, has caused an excessive concentration of income in the highest spheres of the Mexican population, in addition to growing poverty in our country; However, the shortcomings are not distributed equally, since inequality is expressed at the moment in territorial and social inequalities that in turn are a brake on economic growth in the long term. These inequalities can be corroborated by observing the very different levels of development achieved by the entities of our country measured through the Human Development Index. Although Mexico has traditionally been a nation of inequalities, until the mid-eighties of the last century these inequalities had been decreasing; however, the change in the economic model has caused an increase in these inequities. In this sense, the objective of this paper is to know how the concentration of wealth has caused enormous levels of inequality in Mexico. The methodology used is the comparison between the level of Human Development among the federative entities of our country and the evolution of the Gini index to understand how inequality has evolved and in which regions poverty has been concentrated. This reinforces the argument that the neoliberal model is the cause of the growth of poverty and inequality in Mexico.

Keywords: poverty, income, population, inequality, development

\section{Introducción}

La desigualdad es aquel escenario donde existe una falta de equilibrio o equidad entre las partes, siempre y cuando exista un elemento comparativo. Si se considera el factor económico, por lo general, se sustenta en una situación donde el reparto del capital y el disfrute de los factores de bienestar no son equitativos entre las personas. Cuando existe desigualdad en la comunidad se produce la separación de cierto grupo de la sociedad que ha quedado fuera de los beneficios de la riqueza generada por el desarrollo (Torres, 2009, p. 152). ${ }^{2}$ Es decir, la desigualdad social se entiende como aquella situación en la cual los individuos de una comunidad se distinguen de otros por su diferente capacidad de acceso a factores de bienestar humano. Esta diferencia proviene principalmente de la concentración de la riqueza o de la acumulación de propiedades. Por ello la desigualdad limita la capacidad de muchas personas para lograr mejoras en su capital físico, social y humano, necesario para mejorar sus condiciones de vida y bienestar (Esquivel, 2015, p. 5). En un escenario así conviven familias con abundante riqueza, mientras el resto apenas logra sobrevivir.

2. En ese sentido, la marginación también puede funcionar como un indicador de existencia o no de desigualdad en una comunidad. 
Las inequidades económicas se traducen en desigual distribución del ingreso y en acceso diferenciado a educación, salud, vivienda o alimentación; es decir, algunos no tienen la misma posibilidad de obtener los factores básicos del desarrollo que otros (Aguilar, 2016, p. 51). Debido a ello, la desigualdad social, a largo plazo, genera graves problemas en el país, pues provoca la desaceleración del crecimiento económico de las regiones más desarrolladas y, al mismo tiempo, incrementa el rezago de las zonas más atrasadas (Torres \& Delgadillo, 2012, p. 184).

Para el caso de México, la desigualdad se ha expresado tradicionalmente en distorsiones territoriales y sociales que, a su vez, son producto de la excesiva concentración económica (Delgadillo, 2008, p. 73). Sin embargo, esta desigualdad no es solo la desigual distribución del ingreso, sino también incluye la segregación por sexo o género, étnica y por lugar de nacimiento. No obstante, hasta fines de los años setenta del siglo pasado hubo avances importantes en la reducción de las divergencias en nuestro país, medidas a través del índice de Gini (ver figura 1); lamentablemente, con la implementación del modelo neoliberal en 1982 no solo se revierte la tendencia a la homogeneidad, sino también la desigualdad social y territorial comienza a crecer aceleradamente.

En estos momentos, México es uno de los países con mayor desigualdad. Según datos del Comité Oxford para el alivio de la hambruna (OXFAM, su sigla en inglés), nuestro país se ubica entre el $25 \%$ de las naciones con mayor inequidad social en el mundo (Esquivel, 2015, p. 8). El extremo de la desigualdad se observa al comprobar que en 2016 en México había más de 53 millones de pobres (43.6\% de la población), de los cuales 9.4 millones viven en pobreza extrema; ${ }^{3}$ y al mismo tiempo vive aquí Carlos Slim, uno de los hombres más acaudalados del mundo, según la revista Forbes. ${ }^{4}$

3. Véase: Pobreza en México, en Consejo Nacional de Evaluación de la Política de Desarrollo Social, 2017. Disponible en https://www.ConEvAL.org.mx/Medicion/MP/Paginas/Pobreza_2016.aspx.

4. "Con una fortuna de 67.1 mil millones de dólares, Carlos Slim ocupa el lugar número siete entre los más ricos del planeta, y, aunque este año su fortuna creció en casi 23\%, el presidente honorario de América Móvil bajó una posición en el listado de los multimillonarios 2018 de Forbes, dado que en 2017 ocupó el sexto lugar" (Mendoza, 2018, párr. 2). 


\section{Figura 1}

Índice de Gini para México, 1963-2016

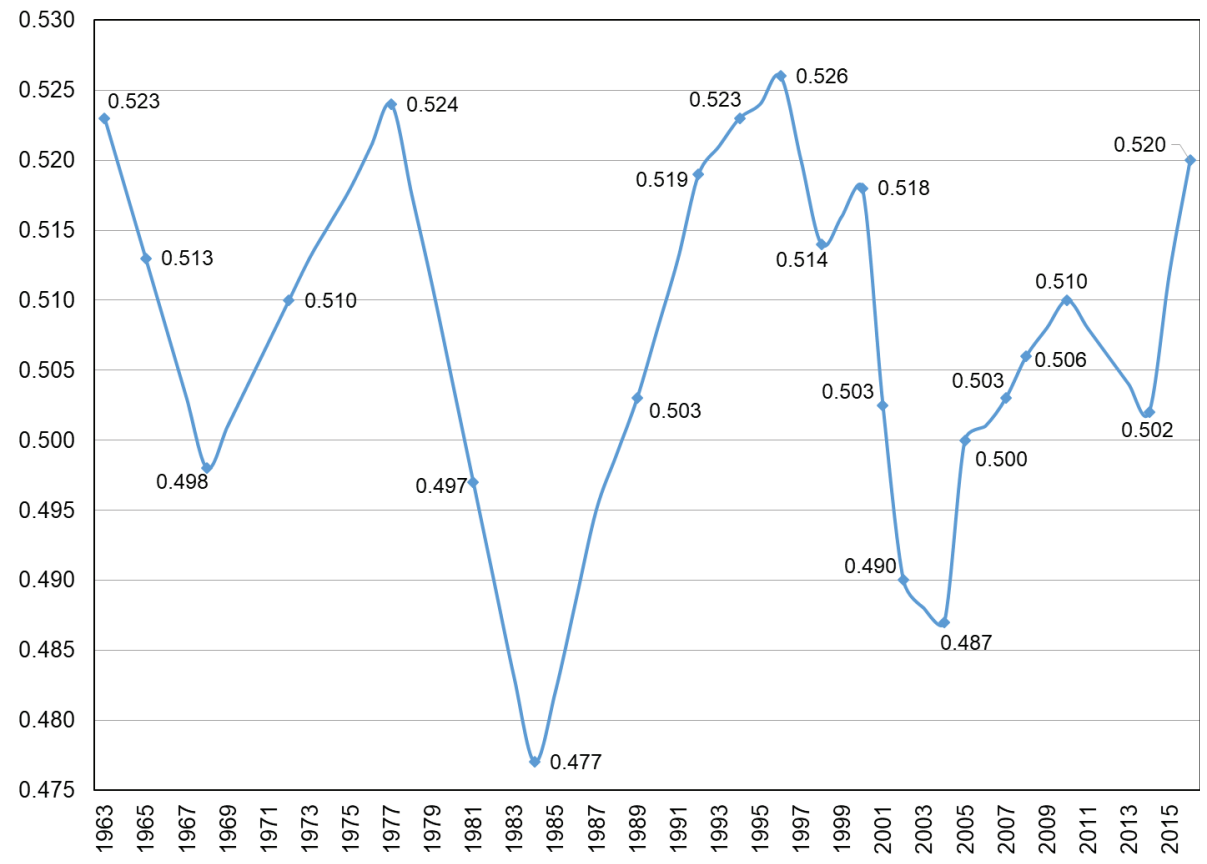

Fuente: Elaboración propia con base en datos del Banco de México de 1963 a 1967, de García, Fuentes y Montes (2012) periodo 1977-2010; para los años 2011-2016, estimaciones propias con base en datos de la Organización para la Cooperación y el Desarrollo Económicos disponible en: https://www.oecd.org/centrodemexico/laocde/.

Por su parte, si bien históricamente México ha sido una nación de claras desigualdades, la apertura comercial en 1986, con el ingreso del país al Acuerdo General sobre Aranceles y Comercio (GATT, su sigla en inglés), favoreció la concentración de la actividad económica en el centro y el norte del país (Lomelí, 2012, p. 135); además, algunas zonas del sureste comenzaron a explotar sus ventajas comparativas, sobre todo las bellezas naturales (como ha sido el caso de la Rivera Maya y la costa de Oaxaca), para atraer turismo internacional. Sin embargo, aquellas entidades mexicanas que no contaban con recursos naturales, infraestructura básica o un mercado potencial en crecimiento no fueron tomadas en cuenta para generar proyectos productivos, por lo que quedaron al margen de la dinámica de las inversiones. 
Algunas entidades del centro occidente, junto con la mayor parte del sur del país, han sido quienes no pudieron insertarse con éxito en el mercado global, por lo que se fueron rezagando, en cuanto a crecimiento y desarrollo económico, del resto del país; de esa manera, ante la apertura económica, la desigualdad territorial continuó ampliándose en México (Esquivel, 2012, p. 142). Más aún, no solo las desigualdades entre las entidades federativas de nuestro país se han ampliado, sino también en su interior las inequidades geográficas y sociales son una constante. Las evidencias empíricas y estadísticas, medidas a través de "la cohesión social" del Consejo Nacional de Evaluación de la Política de Desarrollo Social (CONEVAL), ${ }^{5}$ demuestran que las desigualdades territoriales del país no solo se han mantenido, sino también se han reforzado en los primeros años del actual milenio (Torres \& Delgadillo, 2012, p. 180).

Sobre la base de lo anterior, el objetivo principal de este trabajo es analizar algunos de los factores que han promovido el crecimiento de la pobreza en México, para entender la desigualdad social y territorial en nuestro país. El estudio se basa en la comparación de indicadores oficiales, como el índice de Gini, la distribución del ingreso y el índice de desarrollo humano.

\section{Globalización y desigualdad}

La economía mexicana comenzó su proceso de apertura económica con la implementación del modelo neoliberal al inicio del gobierno de Miguel de la Madrid, en diciembre de 1982. El neoliberalismo tiene como uno de sus postulados básicos la libre competencia y la internacionalización económica. ${ }^{6} \mathrm{Al}$ respecto, la política de apertura comercial de México comenzó formalmente en 1986, con la incorporación de nuestro país al GATT, ${ }^{7}$ mediante lo cual desde ese momento el gobierno mexicano se comprometía a reducir los aranceles y eliminar los precios oficiales de referencia (Clavijo \& Valdivieso, 2000, p. 17).

5. Véase: La cohesión social, en Consejo Nacional de Evaluación de la Política de Desarrollo Social, 2012. Disponible en https://www.coneval.org.mx/Medicion/Paginas/Cohesion_Social.aspx.

6. La internacionalización económica se conoce comúnmente como apertura económica; implica llevar a cabo dos políticas fundamentales: apertura comercial y apertura financiera. Para el caso mexicano, la internacionalización comenzó con la apertura comercial y, posteriormente, con la apertura financiera.

7. No obstante, México había enviado el 16 de enero de 1979 un comunicado donde expresaba su deseo de entablar negociaciones para una eventual adhesión al Acuerdo General (Peña, 1986, p. 44). 
La apertura buscaba intensificar las relaciones económicas y comerciales de nuestro país con el resto del mundo, y fue reforzada en 1989 con un proceso de reforma financiera (apertura financiera), ${ }^{8}$ y en 1992, con la desregulación energética y de inversiones extranjeras (Delgadillo, 2008, p. 73). No obstante, el gobierno neoliberal mexicano amplió su política de apertura comercial con la firma del Tratado de Libre Comercio de América del Norte (TLCAN), que entró en vigor el primero de enero de 1994 y extendía la dependencia económica de México con Estados Unidos.

A partir de ese periodo, el gobierno mexicano le ha dado una enorme importancia a las inversiones extranjeras, y las presenta como sinónimo de crecimiento y desarrollo; sin embargo, todo ello está muy alejado de la realidad, pues no hay que olvidar que toda inversión busca obtener la mayor tasa de ganancia, y por ello seleccionan sus lugares de arribo sobre la base de la subordinación política de los gobiernos locales y, sobre todo, por la existencia de condiciones generales de explotación económica, como bajos salarios, nulas demandas laborales, mínimas condiciones de seguridad de los trabajadores, elevada flexibilidad laboral; en caso de no existir, estas condiciones se convierten en demandas centrales de los grandes inversionistas (Ornelas, 2002, p. 140).

Además, a partir de 1982, el mercado y la libre competencia resurgieron como entes supremos para determinar quién o quiénes sobrevivían, ante el retiro del Estado como agente regulador. Agregado a lo anterior, la apertura comercial ha tenido efectos diferentes en las regiones mexicanas (ChambouxLeroux, 2001, p. 601), y provocó la llegada de cientos de productos a bajo precio (sobre todo orientales y de mala calidad) que inundaron el mercado nacional y dejaron sin ventas a los productores locales, lo cual llevó a la quiebra a miles de pequeños empresarios y a un incremento en el desempleo, con lo que aumentaron la pobreza y la dependencia con el exterior.

Por lo tanto, si bien el incremento en las desigualdades territoriales y sociales en nuestro país es un claro indicador de que no ha existido un avance en el desarrollo, las políticas de mitigación de la pobreza y de promoción de actividades productivas pueden ayudar a reducir esas desigualdades (Sánchez,

8. La reforma financiera de abril de 1989 se sustenta en la liberalización de las tasas de interés bancarias, la sustitución del encaje legal y la desaparición de los cajones de crédito preferencial; ello se vio reforzado con la modificación a la Ley Bancaria en diciembre de 1989 que otorgaba mayor autonomía a los bancos (Sánchez y Sánchez, 2000, p. 691). 
2015, p. 17). No obstante, si bien las inequidades son causadas por el modelo globalizador de la economía, que pone a competir con clara desventaja a pequeños agentes económicos con grandes corporaciones, no solo los factores externos provocaron estas desigualdades: ellas también se deben a una clara falta de estrategia y de acción gubernamental de contención de esos impactos (Torres \& Delgadillo, 2012, p. 180).

En lugar de llevar a cabo políticas de promoción del desarrollo, el gobierno mexicano se ha convertido en un promotor de los inversionistas y ofrece condiciones propicias para las grandes empresas extranjeras para la explotación y apropiación de los recursos naturales de nuestro país, como la política de estricta contención salarial impuesta desde 1982, ${ }^{9}$ la desregulación financiera de 1989, o las privatizaciones, principalmente durante los gobiernos de Miguel de la Madrid (1982-1988) y Carlos Salinas de Gortari (1988-1994). Lo anterior se ha visto reforzado con la urgencia de llevar a cabo un conjunto de reformas en el sexenio de Enrique Peña Nieto (2012-2018): laboral (2012), educativa (2013) y energética (2013), sin importar el bienestar social o el incremento de las desigualdades territoriales y sociales.

\section{Desarrollo humano}

Existen varias formas de estimar en cierto momento el grado de desarrollo alcanzado por una sociedad. Algunos se enfocan principalmente en el ingreso promedio o per capita de la población para señalar si existen las condiciones de bienestar propias a esa población. Sin embargo, tal vez la técnica más reconocida en el mundo, pues se aplica en numerosos países, es el índice de desarrollo humano (IDH), creado por el Programa de las Naciones Unidas para el Desarrollo (PNUD). ${ }^{10}$

El Consejo Nacional de Población (CONAPO) señala que el IDH es una forma de cuantificar si los individuos poseen los recursos suficientes para disfrutar de un nivel de vida digno, cuando disponen de un conjunto de capacidades y oportunidades básicas (Consejo Nacional de Población, 2001, p. 7). Estas capacidades se miden a partir de tres indicadores: 1) gozar de una vida larga y

9. Véase Martín, 2015.

10. "El índice de desarrollo humano (IDH) se creó para hacer hincapié en que la ampliación de las oportunidades de las personas debería ser el criterio más importante para evaluar los resultados en materia de desarrollo" (Informe Nacional de Desarrollo Humano Guatemala, 2016). 
saludable, 2) adquirir conocimientos y 3) participar en la vida de la comunidad (Consejo Nacional de Población, 2001, p. 7).

Sobre esta premisa, el índice de desarrollo humano evalúa el grado de desarrollo de un país sobre la base de las personas y de sus capacidades (Programa de las Naciones Unidas para el Desarrollo, 2015, p. 5), aunque el estudio se puede ampliar a entidades federativas e incluso municipios. Esto permite hacer comparaciones en el nivel de desarrollo alcanzado por varios países y su evolución a través del tiempo. Además, esta diferencia entre el nivel de desarrollo permite conocer la desigualdad existente entre las regiones de un mismo país (Consejo Nacional de Población, 2001, p. 11).

La primera evaluación del IDH en nuestro país con fines comparativos en el ámbito mundial ocurrió en el año 2000; en él se establecía que México, como nación, registraba un índice de 0.791 unidades, por lo que estaba catalogado con un grado medio alto de desarrollo y ocupaba el lugar 51 de entre 162 países catalogados por ese indicador (Consejo Nacional de Población, 2001. pp. 11-12). Sin embargo, debido a las políticas neoliberales, como las ya mencionadas privatizaciones, contención salarial e inestabilidad laboral, así como el estancamiento de la economía mexicana durante la primera década de este milenio, que dejó a mucha gente sin empleo e ingreso, ${ }^{11}$ se acentuó el retroceso del bienestar humano en México, lo cual se vio reflejado en los indicadores de desarrollo.

Al respecto, ya en 2011 México registró un IDH de apenas 0.770, por lo que en ese año se ubicó en la posición 57 en el ámbito mundial (Programa de las Naciones Unidas para el Desarrollo, 2012, p. 7); es decir, en ese periodo nuestro país retrocedió seis lugares. Lamentablemente, el bienestar humano ha continuado su retroceso, pues para 2015 el IDH de México fue de apenas 0.762 unidades, que lo coloca en la posición 73 de entre 187 naciones consideradas para este análisis. ${ }^{12}$ En tan solo quince años el país retrocedió veintidós posiciones y redujo su índice en 29 centésimas, cantidad aparentemente insignificante, pero ello implica que se requieren por lo menos 30 años para recuperar el nivel de desarrollo humano que teníamos en el año 2000.

11. "La primera década del siglo Xxi concluye con un saldo de 2.6 millones de mexicanos en el desempleo y un alza del $333 \%$ en el nivel de desocupación. A quienes en este momento no tienen trabajo se agregan otros 5.4 millones que de plano "desistieron" de buscar porque consideran que no tienen posibilidades de encontrar, de acuerdo con la Encuesta Nacional de Ocupación y Empleo (ENOE)" (González, 2010).

12. Véase: Langner, 2016. 
Sin embargo, se reconoce que durante 50 años sí hubo una reducción en las diferencias estatales del IDH, pues de 1950 al año 2000 hubo avances en la equidad territorial y eso trajo consigo importantes mejoras (López, 2012, p. 118). Por desgracia, ello tuvo un cambio importante, pues desde comienzos de este siglo las desigualdades en el país han sido abrumadoras; por ejemplo, en el año 2000, entre la entidad mejor catalogada de acuerdo con su IDH, el Distrito Federal (hoy Ciudad de México) (0.871) y Chiapas (0.693), que ocupaba el último lugar, había dieciocho centésimas de unidad (Consejo Nacional de Población, 2001, p. 14), cifra no muy significativa, pero que mostraba que, en cuanto a desarrollo humano, había casi veinte años de diferencia entre ambas entidades.

Por desgracia, la desigualdad ha ido en aumento en el país, pues en 2010 la divergencia entre el primer lugar en desarrollo humano y el último fue de veintitrés centésimas (Programa de las Naciones Unidas para el Desarrollo, 2016, p. 113). ${ }^{13}$ En ese año, la Ciudad de México tuvo un IDH de 0.954 y Chiapas de 0.724. Es decir, según el IDH, en esos diez años se amplió la diferencia entre estas dos entidades en cuanto al nivel de bienestar social.

Finalmente, los datos más recientes señalan que entre las entidades que se encuentran en los extremos de bienestar en México existe una diferencia de tan solo dieciséis centésimas. Ello se debe a que la Ciudad de México registró en 2015 un IDH de 0.830, mientras que Chiapas, el último lugar, registra un índice de $0.667 ;^{14}$ no obstante, si bien la diferencia entre ambas se redujo, compararlas es como confrontar el grado de desarrollo entre Andorra (Ciudad de México) y Gabón (Chiapas) (Programa de las Naciones Unidas para el Desarrollo, 2015, pp. 6, 8).

\section{Distribución del ingreso en México}

La pobreza y la desigualdad son dos fenómenos que van de la mano; en México el acelerado crecimiento de las carencias ha provocado que en 2016 casi uno de cada dos mexicanos sea pobre, ${ }^{15}$ ello, entre otros factores, no puede ser más que la expresión clara de la desigualdad. Esto se debe, en gran parte, a la

13. Véase: http://www.mx.undp.org/content/mexico/es/home/library/poverty/informe-nacional-sobredesarrollo-humano-mexico-2016.html.

14. Véase: Xinhua, 2015.

15. Según el ConEVAL, en 2016 la pobreza en México abarcaba al 44\% de la población (Consejo Nacional de Evaluación de la Política de Desarrollo Social, 2017). 
inequitativa distribución del ingreso en nuestro país. Por ejemplo, según el CONEVAL, de acuerdo con la composición del ingreso per capita en México, en 2014 el ingreso promedio en nuestro país fue de tres mil 460 pesos mensuales, mientras que las personas cuyo ingreso se localiza en el primer decil apenas obtuvieron 456 pesos mensuales (tabla 1 ). No obstante, el ingreso de las personas del décimo decil es de trece mil 711 pesos en el mismo periodo (Consejo Nacional de Evaluación de la Política de Desarrollo Social, 2014, p. 112).

Esa clara muestra de desigualdad explica la enorme y creciente pobreza en México, pues el ingreso de las personas ubicadas en el primer decil es tres mil pesos por debajo de la media nacional, mientras aquellos ubicados en el décimo decil reciben más de diez mil pesos por arriba del promedio. Más aun, aquellos que se ubican en los tres deciles superiores tienen ingresos superiores al promedio nacional (tabla 1 ).

\section{Tabla 1}

Ingreso corriente total per capita por deciles en México, 2014

\begin{tabular}{|l|c|c|c|c|c|}
\hline Decil & $\begin{array}{c}\text { Ingreso } \\
\text { promedio }\end{array}$ & $\begin{array}{c}\text { Ingreso } \\
\text { Acumulado }\end{array}$ & $\begin{array}{c}\text { Porcentaje } \\
\text { de acumulado }\end{array}$ & $\begin{array}{c}\text { Diferencia con } \\
\text { el promedio }\end{array}$ & $\begin{array}{c}\text { Múltiplos } \\
\text { del Decil I }\end{array}$ \\
\hline I & 456 & 456 & $1.318 \%$ & $-3,004$ & 1.0 \\
\hline II & 916 & 1,372 & $2.648 \%$ & $-2,244$ & 2.0 \\
\hline III & 1,257 & 2,629 & $3.633 \%$ & $-2,203$ & 2.8 \\
\hline IV & 1,259 & 4,226 & $4.616 \%$ & $-1,863$ & 3.5 \\
\hline V & 1,969 & 6,195 & $5.691 \%$ & $-1,491$ & 4.3 \\
\hline VI & 2,409 & 8,604 & $6.963 \%$ & $-1,051$ & 5.3 \\
\hline VII & 2,984 & 11,588 & $8.625 \%$ & -476 & 6.5 \\
\hline VIII & 3,481 & 15,429 & $11.102 \%$ & 381 & 8.4 \\
\hline IX & 5,458 & 20,887 & $15.775 \%$ & 1,998 & 12.0 \\
\hline X & 13,711 & 34,598 & $39.629 \%$ & 10,251 & 30.1 \\
\hline Promedio & 3,460 & 34,598 & $100.0 \%$ & N.A. & 7.6 \\
\hline
\end{tabular}

Fuente: Elaboración propia basada en datos del Consejo Nacional de Evaluación de la Política de Desarrollo Social, 2014, cuyas estimaciones provienen del MCS-ENIGH 2014. N.A.: No aplica.

A ello se suma que las cifras sobre distribución del ingreso en México en 2014 señalan que las personas del primer decil obtienen apenas el 1.3\% del ingreso promedio, mientras las personas del décimo decil reciben el 39.6\% del promedio nacional de ingresos. Las cifras anteriores explican por qué ya 
en 2014 había en México 55.3 millones de personas en situación de pobreza (Consejo Nacional de Evaluación de la Política de Desarrollo Social, 2014, p. 20 ), cifra que en esos momentos representaba aproximadamente el $46 \%$ del total de los mexicanos. Más aún, las cifras del coneval también señalan que, del total de pobres, 11.4 millones estaban catalogados en pobreza extrema, algo así como el 9.5\% de la población total del país. ${ }^{16}$

Con los datos sobre ingresos se observa la enorme desigualdad en la concentración de ese rubro: en 2014, las personas del décimo decil, correspondientes al $10 \%$ de la población, acaparan el $36.6 \%$ del total de la riqueza generada en el país, y el restante $90 \%$ de los mexicanos se reparte el $60 \%$ de la riqueza (tabla 1). Sin embargo, si a la riqueza generada le sumamos la acumulación de fortuna en periodos pasados, resulta que el $10 \%$ de las personas más acaudaladas en México concentran el $64.4 \%$ de toda la riqueza del país (Esquivel, 2015, p. 7).

Además, de acuerdo con los datos oficiales, la forma en que crece la concentración del ingreso en México es escandalosa, pues las personas ubicadas en el octavo decil consiguen como media 8.4 veces más ingreso que las personas del primer bloque; más aún, la población ubicada en el noveno estrato reciben doce veces el ingreso de las primeras, mientras que los del décimo decil, correspondientes al $10 \%$ de la población, obtienen en promedio 30 veces el ingreso de la población más pobre (tabla 1). Esta desigualdad en la distribución del ingreso ha ido avanzando, pues en 2012 el ingreso promedio de las familias más acaudaladas era 29 veces el correspondiente al de las más pobres (Organización para la Cooperación y el Desarrollo Económico, 2014), mientras que para 2014 esa relación era ya de 30 a uno.

Las políticas implementadas desde la llegada del gobierno de Miguel de la Madrid, de privatizaciones, desregulaciones, apertura comercial y contención salarial (atribuidas al modelo neoliberal), han sido muy exitosas para algunos empresarios mexicanos que, aprovechando el bajísimo costo de la mano de obra y la ausencia de regulaciones, han incrementado enormemente su fortuna. Por ejemplo, durante el periodo 1996-2002 los cuatro multimillonarios mexicanos tenían una riqueza equiparable al $2 \%$ del producto interno bruto

16. Aunque, según cifras oficiales publicadas por el CONEVAL, estos porcentajes de pobreza disminuyeron para 2016, cuando la pobreza abarcaba al 43.6\% de los mexicanos (53.4 millones), de los cuales el 7.6\% vivía en pobreza extrema (9.4 millones de personas) (Consejo Nacional de Evaluación de la Política de Desarrollo Social, 2017). 
(PIB) mexicano. No obstante, para el periodo 2012-2015 esas cuatro personas ya acumulaban una riqueza equiparable al 9\% del PIB nacional (Esquivel, 2015, pp. 18-19).

Los datos anteriores muestran que la concentración de la riqueza en estas personas ha ido de la mano con la reducción del ingreso de las personas más pobres. Según el Instituto Nacional de Estadística y Geografía (INEGI), en 2016 las familias del decil x (los de mayor ingreso) registraron ingresos veintiún veces mayores que las familias del decil I (de menores ingresos), y el $30 \%$ de los hogares con mayores ingresos concentra el 63.3\% de los ingresos, mientras el 30\% de los hogares más pobres apenas recibe el $9 \%$ de la riqueza generada (Albarrán \& Langner, 2017).

Por su parte, el análisis de la participación de las remuneraciones, en la conformación del PIB medido a través del ingreso, muestra que este ha venido a la baja desde 1977; así, mientras en ese año las remuneraciones a asalariados (pago a trabajadores) significaban el 40\% de la riqueza, para 1990 llegaron al 30\%; lamentablemente, en 2012 apenas llegan al 27\% (Esquivel, 2015, p. 26). Es decir, la ya señalada contención salarial ha resultado muy importante en la reducción del ingreso de los trabajadores. ${ }^{17}$

En contraste, en cuanto a la participación de las remuneraciones a capital (utilidades de los empresarios), estas en 1977 representaban el 60\% de la riqueza generada; sin embargo, ya en 1990 los grandes empresarios se apropiaron del $70 \%$ de la fortuna creada en México, para finalmente obtener el 73\% de la riqueza formada en 2012 (Esquivel, 2015, p. 26). Es decir, en los últimos 40 años un mayor número de trabajadores se reparten menor patrimonio (de $40 \%$ a $27 \%$ ), mientras los empresarios incrementaron sus ingresos (60\% a 73\%); además, apenas el 1\% de la población, conformado por el grupo de las personas más acaudaladas en México, se apropia del 21\% de la riqueza generada (Esquivel, 2015, p. 7). Un escenario así solo puede provocar pobreza y desigualdad.

17. El informe presentado por el Centro de Análisis Multidisciplinario de la UNAM señala que en 1987 el salario mínimo alcanzaba para adquirir el 94.3\% de la canasta alimenticia recomendable (CAR), mientras que para el año 2000 apenas alcanzaba para adquirir el 21.87\% de la CAR, y en 2015 solo permitía comprar el $15.45 \%$ de la CAR. Es decir, una pérdida del $79 \%$ del poder adquisitivo en 30 años ("La pérdida acumulada", 2018). 


\section{Pobreza y desigualdad}

La concentración del ingreso en México explica por qué el avance de la pobreza no se ha podido contener, y esta más bien se ha incrementado en los últimos años ${ }^{18}$ mientras en 2008 había 49.5 millones de pobres (el $44.3 \%$ de la población total), en 2010 esa cifra aumentó a 52.8 millones (46.1\%), para alcanzar en 2012 a 53.3 millones de personas (el $45.5 \%$ de la población); finalmente, en 2014 abarcaba ya a 55.3 millones de mexicanos que se encuentran sumidos en la pobreza (el $46.2 \%$ de la población). ${ }^{19}$

Otro dato para tomar en cuenta es que, según el conEvAL, solo el $20.5 \%$ de los mexicanos no sufre de carencias, ni es pobre o vulnerable; es decir, uno de cada cinco mexicanos se puede considerar pudiente, pues su ingreso es suficiente para adquirir todos los bienes y servicios necesarios (Consejo Nacional de Evaluación de la Política de Desarrollo Social, 2014, p. 37). ${ }^{20}$ No obstante, las estadísticas son desalentadoras, pues señalan que cuatro de cada cinco personas registran algún grado de vulnerabilidad. Empero, la distribución de las entidades con porcentajes de población que no es vulnerable es muy heterogénea, así:

1. Existen ocho estados mexicanos donde el promedio de población sin carencias y no vulnerable está por debajo de $13 \%$; es decir, los más pobres (Chiapas, Guerrero, Michoacán, Oaxaca, Puebla, Tlaxcala, Veracruz y Tabasco).

18. Según el CONEVAL, "Una persona se encuentra en situación de pobreza cuando tiene al menos una carencia social (en los seis indicadores de rezago educativo, acceso a servicios de salud, acceso a la seguridad social, calidad y espacios de la vivienda, servicios básicos en la vivienda y acceso a la alimentación) y su ingreso es insuficiente para adquirir los bienes y servicios que requiere para satisfacer sus necesidades alimentarias y no alimentarias" (Consejo Nacional de Evaluación de la Política de Desarrollo Social, 2018).

19. Algo similar ocurrió con la pobreza extrema, la cual también avanzó al mismo ritmo y así, mientras en 2008 el 10.6\% de los mexicanos estaban ubicados en pobreza extrema, en 2010 lo estaba el 11.3\%; mientras que en 2012 era el 9.8, ya para 2014 lo era el 9.5\% (Consejo Nacional de Evaluación de la Política de Desarrollo Social, 2014, p. 20).

20. CONEVAL define la vulnerabilidad por carencias sociales como: "Aquella población que presenta una o más carencias sociales, pero cuyo ingreso es superior a la línea de bienestar". Por su parte la vulnerabilidad por ingresos: "Aquella población que no presenta carencias sociales, pero cuyo ingreso es inferior o igual a la línea de bienestar”, a diferencia de la población pobre, que es aquella cuyo ingreso es insuficiente para adquirir los bienes y servicios que requiere para satisfacer sus necesidades alimentarias y no alimentarias (Consejo Nacional de Evaluación de la Política de Desarrollo Social, 2018). 
2. Por su parte, hay nueve entidades con un rango de entre $14 \%$ y $22 \%$ (Hidalgo, Morelos, Zacatecas, Guanajuato, Estado de México, San Luis Potosí, Yucatán, Campeche y Durango).

3. Además, otros diez estados cuentan con un porcentaje de entre $23 \%$ y 30\% (Sinaloa, Nayarit, Quintana Roo, Querétaro, Colima, Baja California, Tamaulipas, Jalisco, Chihuahua y Baja California Sur).

4. Finalmente, cinco entidades federativas registran un valor entre $31 \%$ y $43 \%$ de población sin carencias, los más ricos (Aguascalientes, Sonora, Coahuila, Ciudad México y Nuevo León).

Lo anterior significa que hay dos grandes extremos, por un lado, en el primer estrato donde se ubican las entidades federativas con mayores carencias de nuestro país, ahí solo una de cada diez personas no sufre de carencias; por consiguiente, las restantes nueve presentan carencias en algún satisfactor. En contraste, en el cuarto bloque, conformado por cinco entidades, tres de cada diez habitantes no sufre de carencia; empero, a pesar de ser las mejores catalogadas, en estas últimas también hay carencias de algún tipo.

En estos momentos, aquellas personas que radican en las entidades del primer bloque tienen el 95\% de posibilidades de tener carencias y ser vulnerables, mientras que, si viven en alguna entidad del cuarto grupo, cuentan con el $65 \%$ de probabilidad de tener carencias. Es decir, aun si se radica en un estado con los mejores indicadores de bienestar, la posibilidad de que una familia caiga en la zona de pobreza es muy alta.

Lo anterior explica gran parte de la desigual distribución de la pobreza: mientras en el primer bloque sobresalen Chiapas y Guerrero, donde apenas el 6\% de su población no sufre de carencias o vulnerabilidad (los peor catalogados), en Nuevo León el $42.5 \%$ de su población está alejada de las carencias y la vulnerabilidad (el mejor clasificado).

Por su parte, al igual que en el ámbito territorial, socialmente la pobreza no se distribuye por igual en México: mientras en promedio uno de cada dos mexicanos se ubica en situación de pobreza, si se analiza la población catalogada como indígena, entonces el promedio aumenta, pues ahí la pobreza abarca a siete de cada diez personas. Es decir, este hecho determina la desigualdad y la discriminación en México que, por el simple hecho de ser indígena, se tiene $70 \%$ de probabilidades de ser pobre. Más aún, mientras el $9.5 \%$ de los mexicanos está catalogado en pobreza extrema, para la población indígena ese porcentaje sube a 38\% (Esquivel, 2015, p. 32); es decir, los indígenas tienen 
cuatro veces más posibilidades de vivir en pobreza extrema que el resto de la población. ${ }^{21}$

Además, mientras el $41.7 \%$ de las personas que habitan zonas urbanas cae en los umbrales de pobreza, en las áreas rurales ese porcentaje se eleva hasta el 61.1\%; en otras palabras, dos de cada cinco habitantes de las ciudades son pobres, mientras que en el ámbito rural tres de cada cinco lo son (Consejo Nacional de Evaluación de la Política de Desarrollo Social, 2014, p. 20).

Como corolario se tiene que la desigualdad social y territorial en México establece que la riqueza y la pobreza no se distribuyen de manera equitativa, pues, como ya se vio, ser indígena o radicar en el medio rural coloca a las personas con un grado mayor de vulnerabilidad que aquellas catalogadas como no indígenas o que viven en ciudades, esto expresa parte de la desigualdad por lugar de residencia. A esto habría que sumarle la discriminación de sexo y género.

\section{Medición de la desigualdad}

En el ámbito mundial, una forma de evaluar la desigualdad en los países es el índice de Gini, ${ }^{22}$ el cual consiste en evaluar la forma en que se distribuye la riqueza entre la sociedad. Ello permite estimar el nivel existente en la concentración de los ingresos entre la población y muestra, por tanto, la inequidad en una sociedad, sobre todo la desigualdad en el ingreso. ${ }^{23}$

Para el caso mexicano, según el Banco de México, en 1963 nuestro país tenía un índice de Gini de 0.523 , por lo que estaba lejos de la equidad social; no obstante, en los siguientes años hubo un ligero avance, pues en 1968 este índice se redujo a 0.498 unidades. Sin embargo, en los siguientes años comenzó un incremento constante de la desigualdad en México, pues en 1977 el país registraba un índice de Gini de 0.524. Para los siguientes años comienza en México un avance en la reducción de las divergencias, pues el índice de Gini

21. "Mientras que el $38 \%$ de la población hablante indígena vive en pobreza extrema, el porcentaje correspondiente para la población total es inferior al 10\%" (Esquivel, 2015, p. 32).

22. Según el Banco Mundial, "El índice de Gini mide hasta qué punto la distribución del ingreso (o, en algunos casos, el gasto de consumo) entre individuos u hogares dentro de una economía se aleja de una distribución perfectamente equitativa. Un índice de Gini de cero representa una equidad perfecta, mientras que un índice de uno representa una absoluta inequidad. Véase: World Bank Group, 2017.

23. Véase: La cohesión social, en Consejo Nacional de Evaluación de la Política de Desarrollo Social, 2012. Disponible en https://www.coneval.org.mx/Medicion/Paginas/Cohesion_Social.aspx. 
comienza a descender para alcanzar un valor de 0.477 en 1984 . No obstante, a partir de la segunda mitad de los años ochenta comienza un incremento constante de la desigualdad, que se mantuvo hasta 1996 cuando el índice de Gini de México alcanzó un máximo de 0.526 (figura 1).

No obstante, las políticas de atención a la pobreza provocaron una mejora temporal, pues los indicadores de igualdad mejoraron, y así el índice de Gini bajó hasta un valor de 0.487 en 2004 . Sin embargo, una vez que alcanzó un mínimo relativo comenzó de nuevo la escalada, y ya en 2016 registró un valor de 0.520. Es decir, después de 50 años nos encontramos ligeramente por debajo de la igualdad registrada en 1963, por lo que luego de las cinco décadas analizadas no se ha avanzado hacia la equidad, ya que los logros alcanzados se revirtieron (figura 1 ).

Lo lamentable del asunto es que la tendencia a la igualdad en México no ha significado una reducción en la pobreza, pues la disminución en el índice de Gini, que supuestamente señala una mejora en la igualdad y una reducción de las inequidades sociales, solo ha mostrado que la pobreza se ha generalizado; esto tiene que ver con que una mayor desigualdad implica una mayor pobreza (Wodon y Yitzhaki, 2002, p. 3). ${ }^{24}$ Por ejemplo, en 1950 se registró el 24\% de pobres (23.9 millones) en México (Székely, 2005, p. 920), ${ }^{25}$ mientras que en 1968 la pobreza ya había alcanzado al 33.1\% de los mexicanos (33.1 millones). Además, en 1984 abarcó al 39.8\% de la población (39.8 millones).

Más aún, en 1994 la pobreza ya afectaba al 51.2\% de la población (66.2 millones) y en el año 2000 al 54\% (54 millones), aunque tuvo una ligera baja en 2004 cuando el $49.6 \%$ de los mexicanos (49.6 millones) era pobre (Székely, 2005, p. 920). Incluso, en 2008 la pobreza se redujo al 44.4\% de la población (49.5 millones), y en 2016 disminuyó hasta abarcar solo al 43.6\% de los mexicanos (53.4 millones); es decir, las variaciones en el índice de Gini se vieron acompañadas por cambios en el porcentaje de pobres, aunque en número absolutos estos han aumentado.

Un dato que confirma lo anterior es que en México el 1\% de las personas con mayor fortuna se apropia en promedio del $22 \%$ de la riqueza que se genera

24. Esto tiene su explicación por el hecho que "para cada determinado nivel de desarrollo económico, una mayor desigualdad implica una mayor pobreza, ya que quienes están en la parte inferior de la distribución de la renta o del consumo obtienen un porcentaje menor de los recursos (Wodon y Yitzhaki, 2002, p. 3).

25. Aquí el autor se refiere a pobres de patrimonio, que es equivalente a lo que el CONEVAL define como pobres (Székely, 2005, p. 920). 
cada año. Este acaparamiento de capital es tal que México se coloca como el país con mayor desigualdad en la distribución del ingreso, muy alejado de los países con mayor equidad, como Dinamarca y Suecia, donde el 1\% de los ciudadanos más acaudalados se apoderan únicamente del 7\% de la riqueza que se genera en sus países (Esquivel, 2015, p. 15).

Finalmente, una estrategia de reducción de las disparidades sociales en México debe partir de una mejora en la cohesión social y del compromiso de toda la sociedad por reconocer que todos los seres humanos tenemos derecho a la seguridad alimentaria y social, a una educación de calidad y a un trabajo e ingreso dignos. El gobierno tiene la tarea de llevar a cabo políticas de contención de la pobreza y de mantener la paz social a fin de revertir las tendencias de la desigualdad.

\section{Conclusiones}

Si bien México venía de un avance importante en la reducción de las desigualdades desde mediados del siglo pasado y hasta la década de los ochenta, la llegada del neoliberalismo provocó que se revertiera la situación y desde los años noventa del siglo xx hubo un importante crecimiento en la inequidad. Ello se debió a que los beneficios del desarrollo no se repartieron de manera equitativa, y ello ha provocado desigualdades: en lo social, concentrando la riqueza en los estratos que ya contaban con los mayores ingresos; en lo territorial, mejorando el bienestar solo en aquellas entidades con mayores porcentajes de urbanización.

Por su parte, las áreas rurales y la población indígena han tendido a concentrar la pobreza y las carencias. Así, mientras que en promedio uno de cada dos mexicanos es pobre, para las áreas rurales la media es de dos de cada tres. Además, si en estos momentos uno de cada diez mexicanos se ubica en la línea de pobreza extrema, para las zonas rurales uno de cada tres personas lo es. Es decir, la desigualdad como expresión de la discriminación racial y de residencia.

En los últimos años, el menor nivel de desigualdad entre la población mexicana, medido por el índice de Gini, se alcanzó en 1984 (0.447), mientras que para 1996 la inequidad social alcanzó su máximo nivel (0.526). De esta manera, los logros alcanzados hasta principios de los ochenta del siglo pasado se han revertido, y ahora México es uno de los países más desiguales del mundo. Esa excesiva concentración de la riqueza ha derivado en que el 
ingreso promedio de las familias con mayor fortuna, correspondiente al 10\% de la población mexicana, sea 30 veces el ingreso que reciben las familias más pobres del país.

En ese sentido, las políticas que buscan mejorar la competitividad empresarial basadas en el bajo costo de la mano de obra han provocado un grave deterioro salarial y el aumento en la informalidad laboral; con ello han arrojado a millones de personas al desempleo y el subempleo, con el consecuente crecimiento de la pobreza, la desigualdad y la migración laboral hacia Estados Unidos.

\section{Referencias}

Aguilar, T. (2016). Desigualdad y marginación en Chiapas y Michoacán. En L. Rodríguez Castillo (Comp.), Acción pública y política pública para el desarrollo (pp. 47-80). San Cristóbal de Las Casas: CIMSUR / UNAM.

Albarrán, E., \& Langner, A. (2017, 28 de agosto). Familias ricas ganan 21 veces más que las pobres. El Economista. Recuperado de https://www. eleconomista.com.mx/economia/Familias-ricas-ganan-21-veces-masque-las-pobres-20170828-0089.html

Chamboux-Leroux, J. I. (2001). Efectos de la apertura comercial en las regiones y la localización industrial en México. Revista Comercio Exterior, 51 (7), 600-609. Recuperado de http://revistas.bancomext.gob.mx/rce/ magazines/34/2/RCE.pdf

Clavijo, F., \& Valdivieso, S. (2000). Reformas estructurales y política macroeconómica: el caso de México 1982-1999. Recuperado de https://repositorio. cepal.org/bitstream/handle/11362/7602/1/S2000584_es.pdf

Consejo Nacional de Evaluación de la Política de Desarrollo Social. (2012). La Cohesión Social. Recuperado de https://www.Coneval.org.mx/Medicion/ Paginas/Cohesion_Social.aspx

Consejo Nacional de Evaluación de la Política de Desarrollo Social (coneVAL). (2014). Informe de pobreza en México 2014. Recuperado de https:// www.coneval.org.mx/InformesPublicaciones/Documents/Informe-pobreza-Mexico-2014.pdf\#search=Informe\%20de\%20pobreza\%20en\%20 M\%С3\%A9xico\%202014

Consejo Nacional de Evaluación de la Política de Desarrollo Social (CONEVAL). (2017). Pobreza en México. Recuperado de https://www.Coneval.org.mx/ Medicion/MP/Paginas/Pobreza_2016.aspx 
Consejo Nacional de Evaluación de la Política de Desarrollo Social (CONEVAL). (2018). Glosario. Recuperado de https://www.coneval.org.mx/Medicion/ Paginas/Glosario.aspx

Consejo Nacional de Población (CONAPO). (2001). Índices de desarrollo humano, 2000, México: CONAPO. Recuperado de http://www.conapo.gob.mx/work/ models/cONAPO/Resource/211/1/images/dh_Indices.pdf

Delgadillo, J. (2008). Desigualdades territoriales en México derivadas del tratado de libre comercio de América del Norte. Revista EURE - Revista Latinoamericana de Estudios Urbano Regionales, 34(101), 71-98. Recuperado de http://www.eure.cl/index.php/eure/article/view/1336

Esquivel, G. (2015). Desigualdad extrema en México, México, Oxfam México. Recuperado de https://www.oxfammexico.org/sites/default/files/desigualdadextrema_informe.pdf

Esquivel, G. (2012). La desigualdad en las entidades federativas. En G. Meixueiro, S. Moreno \& C. Martínez (Coords.), Desarrollo regional y competitividad en México (pp. 141-155). México: Centro de Estudios Sociales y de Opinión Pública. Recuperado de file://C:/Users/Nora/Downloads/ Desarrollo-regional-competitividad-mexico.pdf

García, A., Fuentes, N., \& Montes, O. (2012). Desigualdad y polarización del ingreso en México: 1980-2008. Política y Cultura, (37), 285-310.

González, S. G. (2010, 26 de diciembre). Subió 333\% el desempleo en 10 años de gobiernos del PAN, señala Inegi. La Jornada. Recuperado de http:// www.jornada.unam.mx/2010/12/26/economia/019n1eco

Informe Nacional de Desarrollo Humano Guatemala. (2016). Índice de Desarrollo Humano. Recuperado de http://desarrollohumano.org.gt/desarrollohumano/calculo-de-idh/

La pérdida acumulada del poder adquisitivo en los últimos 30 años es de 80 por ciento: expertos de la UNAM. (2018, 12 de enero). Boletín UNAMDGCS, 016. Recuperado de http://www.dgcs.unam.mx/boletin/bdboletin/2018_016.html

Langner, A. (2016, 14 de enero). Avance económico de México se diluye por su bajo IDH. El Economista. Recuperado de https://www.eleconomista. com.mx/politica/Avance-economico-de-Mexico-se-diluye-por-su-bajoIDH-20160114-0041.html

Lomelí, L. (2012). Potencialidades de desarrollo económico de las regiones de México. En G. Meixueiro, S. Moreno \& C. Martínez (Coords.), Desarrollo regional y competitividad en México (pp. 131-139). México: Centro de 
Estudios Sociales y de Opinión Pública. Recuperado de file://C:/Users/ Nora/Downloads/Desarrollo-regional-competitividad-mexico.pdf López, L. F. (2012). Evolución del desarrollo humano en México. En G. Meixueiro, S. Moreno \& C. Martínez (Coords.), Desarrollo regional y competitividad en México (pp. 117-130). México: Centro de Estudios Sociales y de Opinión Pública. Recuperado de file://C:/Users/Nora/Downloads/ Desarrollo-regional-competitividad-mexico.pdf

Martín, R. (2015, 11 de enero). 38 años de contención salarial. El Economista. Recuperado de https://www.eleconomista.com.mx/opinion/38-anos-decontencion-salarial-20150112-0001.html

Mendoza, V. (2018, 6 de marzo). La (itinerante) fortuna de Carlos Slim. Forbes México. Recuperado de https://www.forbes.com.mx/la-itinerantefortuna-de-carlos-slim/

Organization for Economic Cooperation and Development (OECD). (2014). Society at a Glance 2014: OECD Social Indicators. Recuperado de http://www. oecd.org/social/societyataglance.htm doi: 10.1787/soc_glance-2014-en Ornelas, J. D. (2002). El Plan Puebla-Panamá y la globalización neoliberal. Aportes: Revista de la Facultad de Economía-BUAP, VII (21), 137-155.

Peña, R. (1986). Ventajas y desventajas del ingreso de México al GATT. Comercio Exterior, 36(1), 33-45. Disponible en http://revistas.bancomext. gob.mx/rce/magazines/206/4/RCE4.pdf

Programa de las Naciones Unidas para el Desarrollo (PNUD). (2016). Informe sobre Desarrollo Humano México 2016. Desigualdad y movilidad. México: Programa de las Naciones Unidas para el Desarrollo. Recuperado de http://www.mx.undp.org/content/dam/mexico/docs/Publicaciones/ PublicacionesReduccionPobreza/InformesDesarrolloHumano/idhmovilidadsocial2016/PNUD\%20IDH2016.pdf

Programa de las Naciones Unidas para el Desarrollo (PNUD). (2015). Índice de Desarrollo Humano para las entidades federativas, México 2015. Avance continuo, diferencias persistentes. México: Programa de las Naciones Unidas para el Desarrollo. Recuperado de http://www.mx.undp.org/content/ dam/mexico/docs/Publicaciones/PublicacionesReduccionPobreza/InformesDesarrolloHumano/PNUD_boletinIDH.pdf

Programa de las Naciones Unidas para el Desarrollo (PNUD). (2012). El Índice de Desarrollo Humano en México: cambios metodológicos e información para las entidades federativas, México: Programa de las Naciones Unidas para el 
Desarrollo. Recuperado de http://www.cinu.mx/minisitio/indice_de_desarrollo/El_IDH_en_Mexico.pdf

Sánchez, A. A. (2015). Las teorías de desarrollo regional. En Autor (Coord.), El desarrollo económico de las regiones medias de México (pp.17-26). México: IIEc-UNAM.

Sánchez, R. T., \& Sánchez, A. D. (agosto, 2000). Desregulación y apertura del sector financiero mexicano. Comercio Exterior, 50(8), 686-697.

Székely, M. (2005). Pobreza y desigualdad en México entre 1950 y 2004. El Trimestre Económico, LXXII(288), pp. 913-931.

Torres, F. T. (Coord). (2009). Técnicas para el análisis regional. Desarrollo y aplicaciones. México: Trillas.

Torres, F. T., \& Delgadillo, J. M. (2012). Nueva encrucijada del desarrollo regional en México. En J. L. Calva (Coord.), Desarrollo regional y urbano (pp. 179-200). México: Juan Pablo Editor-Consejo Nacional de Universitarios.

Wodon, Q., \& Yitzhaki, S. (2002). Desigualdad y bienestar social. Nueva York: Banco Mundial. Recuperado de http://siteresources.worldbank.org/INTPRS1/ Resources/383606-1205334112622/4768783-1205337105916/11101_ ineq_sp.pdf

World Bank Group. (2017). Coeficiente de Gini. Recuperado de http://pubdocs. worldbank.org/en/137751424971740500/NT-Desigualdad.pdf

Xinhua. (2015, 4 de marzo). PNUD: el mayor índice de desarrollo humano en México lo tiene Distrito Federal. América Economía. Recuperado de https://www.americaeconomia.com/economia-mercados/finanzas/pnudel-mayor-indice-de-desarrollo-humano-en-mexico-lo-tiene-distrito-fed 